\title{
Avaliação do aquecimento de amostras de aço imersas em plasma
}

\author{
Evaluation of heat transfer in steel \\ samples immersed in plasma
}

\author{
Otávio Henrique de Andrade Disconzi ${ }^{1}$, Diego Michael Cornelius dos Santos ${ }^{1}$, \\ Bruno Cuchi Bordignon ${ }^{1}$, Júlia Beltrame Bisogno ${ }^{1}$, \\ Inácio da Fontoura Limberger ${ }^{1}$ Natália de Freitas Daudt ${ }^{1}$
}

\begin{abstract}
${ }^{1}$ Universidade Federal de Santa Maria (UFSM), Departamento de Engenharia Mecânica, Grupo de Pesquisa em Tecnologia e Mecânica dos Materiais (GMat), Avenida Roraima, 1000, CEP: 97105-900, Santa Maria, RS, Brasil. E-mail: otavioandrade1@ @otmail.com, diegomichael.santos@gmail.com, brunocuchibordignon@gmail.com, jbeltramebisogno@gmail.com, oicanis@gmail.com, natalia.daudt@ufsm.br.
\end{abstract}

\section{RESUMO}

Processos assistidos por plasma tem diversas aplicações industrias como a nitretação de aços, sinterização, deposição de filmes finos e produção de semicondutores. A taxa de aquecimento, transferência de calor e temperatura têm um papel fundamental nas propriedades dos materiais imersos em um plasma. Contudo, um dos desafios do processamento de materiais por plasma é medir com precisão a transferência de calor e a temperatura, particularmente em regiões especificas da amostra, uma vez que pode haver a presença picos térmicos, aumentando localmente a temperatura. Além disso, há diversos parâmetros como a composição da atmosfera, potência, pressão e a composição da amostra que podem afetar a difusão, o transporte de massa e a taxa de aquecimento no plasma. Neste contexto, o objetivo deste estudo é avaliar a homogeneidade do aquecimento de amostras de aços revenidas em plasma de argônio e comparar com o aquecimento em forno resistivo. Para este propósito, a microestrutura e a dureza de amostras revenidas em plasma foram comparadas às amostras revenidas em forno resistivo. Desse modo, foi possível determinar a temperatura equivalente de um sólido imerso em plasma. Tendo em vista o grande interesse industrial em processos de nitretação a plasma, neste estudo o aquecimento de amostras de aços imersas em plasma foi avaliado em um reator típico de nitretação a plasma. Para tal, amostras de aço SAE 1045 e 8640 foram temperadas e em seguida revenidas em plasma. As amostras revenidas em plasma mostraram uma maior perda de dureza para o tratamento na mesma temperatura (medida no porta-amostra) do que as amostras revenidas convencionalmente. Este resultado foi relacionado aos picos térmicos durante o aquecimento a plasma. Um modelo matemático para determinar a temperatura equivalente durante o revenimento a plasma foi proposto. Este modelo poderá ser aplicado para desenvolver estratégias para otimizar os parâmetros do plasma, visando melhorar as propriedades dos materiais.

Palavras-chave: Aquecimento em plasma, Tratamento térmico, Transferência de calor, Microdureza, Aço ao carbono.

\section{ABSTRACT}

Plasma-assisted processes have several industrial applications such as steel nitriding, sintering, thin film deposition and semiconductor production. Heating rate, heat transfer and temperature play a key role in the properties of materials immersed in a plasma. However, one of the challenges of plasma processing of materials it is to accurately measure the heat transfer and the temperature, particularly in a specific region of the sample, as there may be thermal spikes, raising locally the temperature. Furthermore, there are several parameters such as plasma atmosphere composition, power, pressure and sample composition that can affect diffusion, mass transport and plasma heating rate. Thus, the aim of this study is to evaluate the heating homogeneity of tempered steel samples in argon plasma and compare it to heating in a resistive furnace. For this purpose, microstructure and hardness of plasma tempered samples were compared to samples conventionally tempered 
in a resistive furnace, so that the equivalent temperature of a solid immersed in a plasma could be determined. In view of the great industrial interest on plasma nitring process, in this study the heating of steel samples immersed in plasma in a typical plasma nitriding chamber was evaluated. For that samples made of SAE 1045 and SAE 8640 steel were quenched and then tempered in plasma. The plasma tempered samples showed a much smaller hardness for treatment at same measured temperature than conventionally tempered samples. This result was related to thermal spikes during plasma heating. A mathematical model to set the equivalent temperature during plasma tempering was proposed. From this model it is expected to develop strategies to optimize plasma parameters, aiming on improving materials properties.

Keywords: plasma heating, heat treatment, heat transfer, microhardness, carbon steel.

\section{INTRODUÇÃO}

O plasma tem se mostrado uma fonte energética importante no processamento de materiais, devido à sua grande versatilidade [1], permitindo a modificação da superfície de materiais poliméricos [2], tratamentos termoquímicos [3], produção de semicondutores [4], obtenção de revestimentos de diferentes materiais como carbono tipo diamante [5], nanotubos de carbono [6] e TiN [7].

O plasma pode ser definido como uma mistura de gás, elétrons livres e íons, os quais interagem entre si. O plasma, utilizado no processamento de materiais é comumente obtido através da aplicação de uma diferença de potencial entre dois eletrodos inseridos em um reator fechado que contém gás em seu interior [8]. A diferença de potencial aplicada gera um campo elétrico, provocando acelerações de elétrons livres. As colisões entre os elétrons livres e as partículas do plasma resultam na ionização parcial do gás. O grau de ionização do plasma dependerá da energia cinética dos elétrons que por sua vez está relacionada com o campo elétrico aplicado. De acordo com seu equilíbrio termodinâmico, o plasma pode ser classificado em térmico e não térmico. No plasma térmico, as espécies estão equilíbrio térmico, ou seja, a temperatura dos elétrons livres, íons e partículas é a mesma. Enquanto no plasma não-térmico, temperatura dos elétrons é muito maior que das demais espécies, o que faz com que a medição local de temperatura no plasma seja um desafio $[1,9]$.

O plasma térmico, resultado de uma ionização quase completa dos gases, é frequentemente utilizado nos processos de aspersão térmica a plasma para a obtenção de revestimentos cerâmicos resistentes à corrosão e à temperatura [10]. O plasma não-térmico, resultado de um baixo grau de ionização dos gases, muitas vezes gerado em reatores de baixa pressão, é amplamente utilizado para modificar a estrutura superficial dos materiais poliméricos, nitretação iônica e deposição de filmes finos através de processos de deposição física de vapor $[9,10]$. No caso do plasma não-térmico utilizado nos tratamentos de nitretação iônica, o plasma é gerado a partir da aplicação de uma diferença de potencial entre o porta-amostra (cátodo) e a carcaça do reator (ânodo), nesse caso íons carregados positivamente entre eles o N+ são acelerados em direção à superfície da peça a ser nitretada, posicionada sob o porta-amostra. A energia resultante do bombardeamento iônico é suficiente para realizar o aquecimento das peças, intensificando o processo de difusão [11]. As características do plasma gerado neste processo dependem de algumas variáveis como: diferença de potencial aplicada, tempo de exposição do material [12], composição da mistura dos gases [13], geometria dos eletrodos [14] e pressão $[9,15]$.

Em sua totalidade, a transferência de calor no plasma é influenciada pela radiação e pela colisão de íons na amostra. Através da radiação, a energia é transferida de maneira homogênea e equidistante à superfície, e pelas colisões de íons a energia é transferida para as microrregiões. O mecanismo de radiação é o resultado da energia cinética e potencial das partículas, atuando de forma considerável apenas em altas temperaturas [16]. Desta maneira, o aquecimento do substrato em sua maior parte, é consequência de reações exotérmicas que ocorrem na superfície, devido a colisão de íons. As colisões entre os íons do plasma e uma determinada região de um sólido são capazes de produzir elevadas taxas de energia no sólido, causando assim um elevado grau de aquecimento localizado que pode resultar em uma modificação na microestrutura do material, promovendo alterações na dureza e na resistência mecânica [17]. Este fato pode ocasionar um gradiente térmico, que tem origem na superfície, direcionando-se até o centro da amostra, visto que a taxa de aquecimento da superfície é maior do que a taxa de transferência de calor por condução existente no interior do material [16]. Por esse motivo é difícil medir com precisão a temperatura de um sólido imerso no plasma, e as medidas de temperatura devem ser analisadas com cuidado, já que a temperatura média pode ser consideravelmente inferior ao pico de temperatura. Apesar da presença de gradiente térmicos em sólidos aquecidos em plasma ser um fenômeno conhecido na literatura há poucos os trabalhos dedicados a medi-lo [18-22].

Além do gradiente térmico gerado pelos picos de temperatura, as espécies ativas do plasma também têm um efeito na microestrutura dos sólidos aquecidos no plasma. Por exemplo, um maior crescimento de grão (uma ordem de magnitude maior) e uma maior densificação foram observados em amostras metálicas 
sinterizadas em plasma quando comparadas a amostras sinterizadas em forno resistivo [23-25]. O maior crescimento de grão foi associado à presença de espécies de hidrogênio no plasma que removem impurezas do contorno de grão facilitando o crescimento do grão [23], e ao provável aumento na taxa de difusão e transporte de massa que acelerou a densificação das amostras [24]. Além disso, a combinação de picos de temperatura e espécies de hidrogênio permitiu a sinterização de partículas metálicas imersas em plasma em temperaturas muito menores do que as convencionalmente utilizadas na sinterização resistiva [26- 28]. Logo, conclui-se que o plasma promove os processos termicamente ativados.

Os processos assistidos por plasma são complexos, visto que uma série de parâmetros estão envolvidos. Todavia, as modificações desses parâmetros dão uma grande versatilidade a esses processos permitindo diversas aplicações em engenharia, tais como: tratamentos termoquímicos (nitretação iônica), sinterização de ligas, deposição de filmes finos, polimerização e o desenvolvimento de novos materiais [21]. Tendo em vista que a temperatura, bem como a taxa de difusão, transferência de calor e massa têm um papel fundamental nos processos assistidos por plasma, em especial nas propriedades mecânicas dos aços submetidos a nitretação iônica; este trabalho teve como objetivo avaliar o aquecimento de amostras de aço imersas em plasma em um reator de nitretação iônica. Para tal, foi realizado um estudo comparativo entre o aquecimento de amostras de aço durante revenimento em plasma e em forno resistivo. A homogeneidade do aquecimento das amostras, foi avaliada através de testes de microdureza e microscopia. Um modelo matemático para determinação da temperatura equivalente em sólidos imersos em plasma em um reator de nitretação foi proposto. Como materiais de partida foram utilizados aços SAE 1045 e 8640, por serem materiais muito utilizados para fazer componentes mecânicos que comumente passam pelo processo de têmpera e revenido e por apresentarem uma boa sensibilidade a pequenas variações nas temperaturas de revenimento.

\section{MATERIAIS E MÉTODOS}

\subsection{Materiais de Partida}

Para a realização deste estudo, foram utilizadas barras dos aços SAE 1045 e SAE 8640 com 18 mm de diâmetro, da fabricante Gerdau, com composição química média em massa descrita pelo fabricante de 0,43$0,50 \%$ de $\mathrm{C}, 0,60-0,90 \%$ de $\mathrm{Mn}, \leq 0,04 \%$ de $\mathrm{P}$ e $\leq 0,05 \%$ de $\mathrm{S}$ para o aço SAE 1045 e de $0,38-0,43 \%$ de $\mathrm{C}$, 0,75-1,00\% de Mn, 0,40-0,60\% de Cr, 0,40-0,70\% de Ni e 0,15-0,25\% de Mo para o aço SAE 8640. De cada uma das barras foram cortados 16 cilindros com $18 \mathrm{~mm}$ de diâmetro e $20 \mathrm{~mm}$ de altura. As amostras de ambos os aços foram temperadas, para tal, as amostras foram aquecidas em um forno resistivo até $845^{\circ} \mathrm{C}$ e mantidas nessa temperatura por 30 minutos, e então temperadas em uma mistura de água e gelo a temperatura de $0^{\circ} \mathrm{C}$. Posteriormente, duas amostras de cada tipo de aço foram aquecidas no mesmo forno resistivo até a temperatura de revenimento com uma taxa de aquecimento de cerca de $10{ }^{\circ} \mathrm{C} / \mathrm{min}$ e mantidas nesta temperatura por 30 minutos, este procedimento foi repetido para o revenimento a $200{ }^{\circ} \mathrm{C}, 300{ }^{\circ} \mathrm{C}, 400{ }^{\circ} \mathrm{C}, 500$ ${ }^{\circ} \mathrm{C}$ e $600{ }^{\circ} \mathrm{C}$.

\subsection{Revenimento em plasma}

As amostras dos aços SAE 1045 e SAE 8640, previamente temperadas, foram revenidas em plasma, utilizando o reator de nitretação iônica especialmente construído para o Laboratório de Superfícies da UFSM. Neste reator, o plasma é gerado em uma câmara de vácuo, a partir da aplicação de uma diferença de potencial entre o porta-amostra (cátodo) e as paredes do reator (ânodo), conforme ilustrado na Figura 1. 


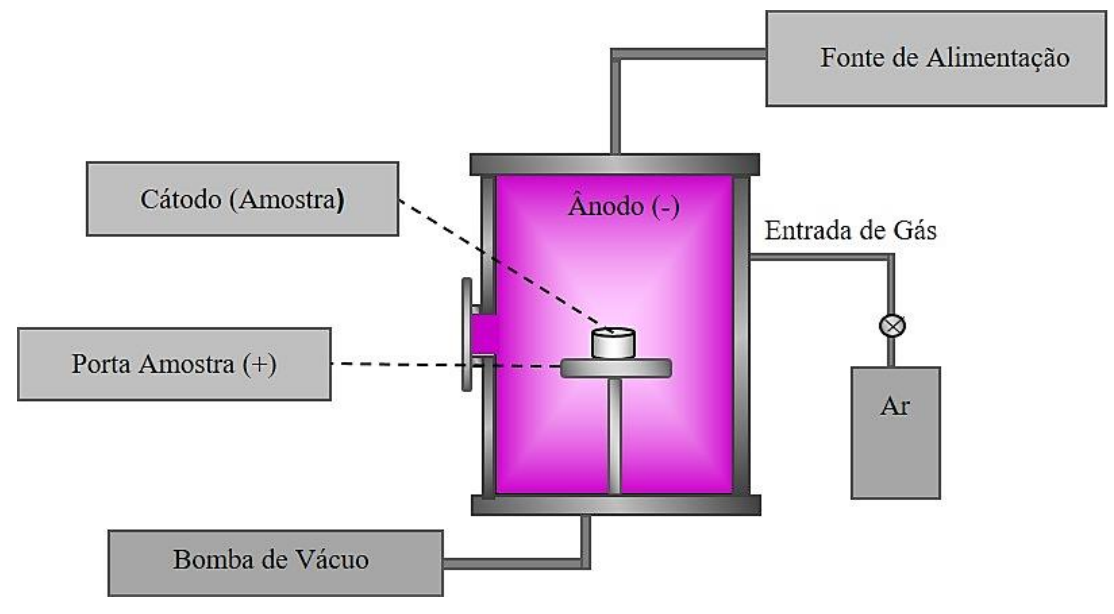

Figura 1: Representação esquemática do sistema de nitretação iônica.

Os tratamentos de revenimento a plasma foram feitos nas amostras de ambos os aços previamente temperados. Para isso foram utilizadas correntes de $100 \mathrm{~mA}, 200 \mathrm{~mA}$ e $300 \mathrm{~mA}$, sendo que o potencial elétrico para cada um dos casos foi ajustado para obter a corrente pré-determinada. Em cada uma destas correntes foram revenidas duas amostras de cada tipo de aço. A pressão na câmara de vácuo durante os tratamentos de revenimento foi mantida em $100 \mathrm{~Pa}$ através da adição de um fluxo controlado de argônio. Assumiu-se a partir de experimentos prévios que após $30 \mathrm{~min}$ na corrente pré-determinada, as amostras atingem a temperatura de revenimento, assim sendo após atingir a temperatura de revenimento as amostras foram mantidas por mais 30 minutos sob corrente constante para garantir uma temperatura uniforme no porta-amostra. Ao final do tratamento, com o auxílio de um pirômetro, realizou-se a leitura da temperatura no porta-amostra.

\subsection{Caracterização das amostras}

As amostras revenidas foram avaliadas pelo teste de microdureza Vickers, utilizando um microdurômetro da marca Schimadzu, modelo HMV-2. Para a medição de dureza, as amostras foram seccionadas transversalmente, em uma cortadeira de disco refrigerado, e na sequência foram polidas. A medição da dureza foi realizada na seção transversal das amostras em intervalos de $2 \mathrm{~mm}$, partindo da superfície da amostra, percorrendo todo o seu diâmetro, como pode ser observado na Figura 2. Apenas os resultados de dureza da superfície até o centro da amostra serão apresentados. Para realização deste ensaio uma carga de 9,807 N foi aplicada durante 10s, seguindo a NBR ISO 6507-1:2019 [29].

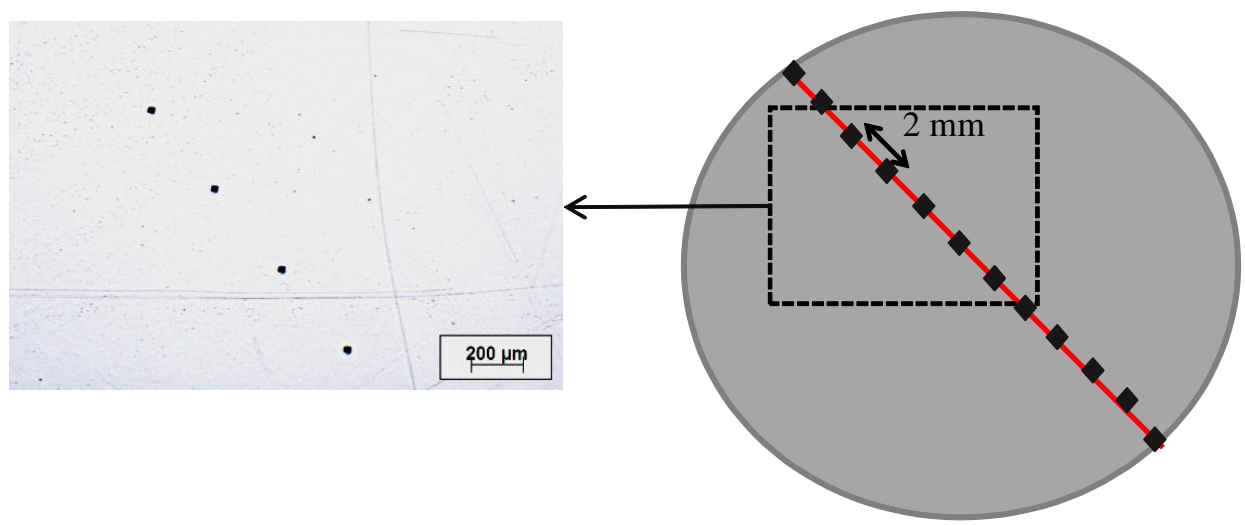

Figura 2: Esquema da medição de dureza através da seção transversal das amostras.

Após a realização dos testes de dureza, as amostras foram novamente polidas e submetidas a um ataque químico, com uma solução de $2 \%$ de ácido nítrico (Nital 2\%) para a realização da análise microestrutural. A microestrutura das amostras foi analisada utilizando um microscópio eletrônico de varredura (Jeol JSM 6360). 


\subsection{Determinação do modelo matemático para verificação de temperaturas}

Os valores de dureza Vickers das amostras revenidas no plasma foram comparados com as obtidas nas amostras revenidas em forno resistivo e a partir dessa comparação um modelo matemático para determinar a temperatura equivalente das amostras tratadas no plasma foi proposto. Para a determinação do modelo matemático, assumiu-se que para obter um valor de dureza igual nas amostras revenidas, uma temperatura equivalente à do forno resistivo deve ser aplicada no plasma. A partir dos dados de dureza média e temperatura foi proposta uma função polinomial de segunda ordem do tipo $a x^{2}+b x+c$, para determinação da temperatura de revenimento em função da dureza no forno resistivo. Essa função foi então aplicada para determinação da temperatura equivalente durante o revenimento em plasma.

\section{RESULTADOS E DISCUSSÕES}

A microestrutura e os valores de dureza obtidos após o revenimento em forno resistivo das amostras de referência do aço SAE 1045 estão apresentados na Figura 3 e Tabela 1, respectivamente. O aço SAE 1045 temperado em água apresentou a formação de martensita com estrutura acicular bem definida (Figura 3 a). $\mathrm{O}$ revenimento a $200{ }^{\circ} \mathrm{C}$ (Figura 3 b) não resultou em mudanças significativas na microestrutura do SAE 1045, houve apenas leve diminuição na definição da estrutura acicular da martensita, o que vai de encontro com valores de dureza que praticamente não foram alterados. Ao aumentar a temperatura de revenimento para 400 ${ }^{\circ} \mathrm{C}$ (Figura $3 \mathrm{c}$ ) há formação de uma martensita revenida com estrutura acicular menos definida e com a presença de alguns poucos carbonetos. Já nas amostras revenida a $600{ }^{\circ} \mathrm{C}$ (Figura 3 d), observa-se uma martensita revenida com estrutura pouco definida, e uma grande quantidade de carbonetos (região mais clara). Ao analisar os valores de dureza pode-se assumir que há recristalização da martensita com a diminuição considerável das discordâncias nas amostras revenidas acima de $300{ }^{\circ} \mathrm{C}$ e partir de $400{ }^{\circ} \mathrm{C}$ há o início da esferoidização dos carboneto de ferro, o qual se torna mais pronunciado na amostra revenida a 600 ${ }^{\circ} \mathrm{C}$, na qual houve a formação da esferoidita (Figura $3 \mathrm{~d}$ ). Assim sendo, nota-se que conforme a temperatura de revenimento aumenta, as mudanças microestruturais se tornam mais expressivas, o que está de acordo com o comportamento previsto pela literatura [30, 31, 33].

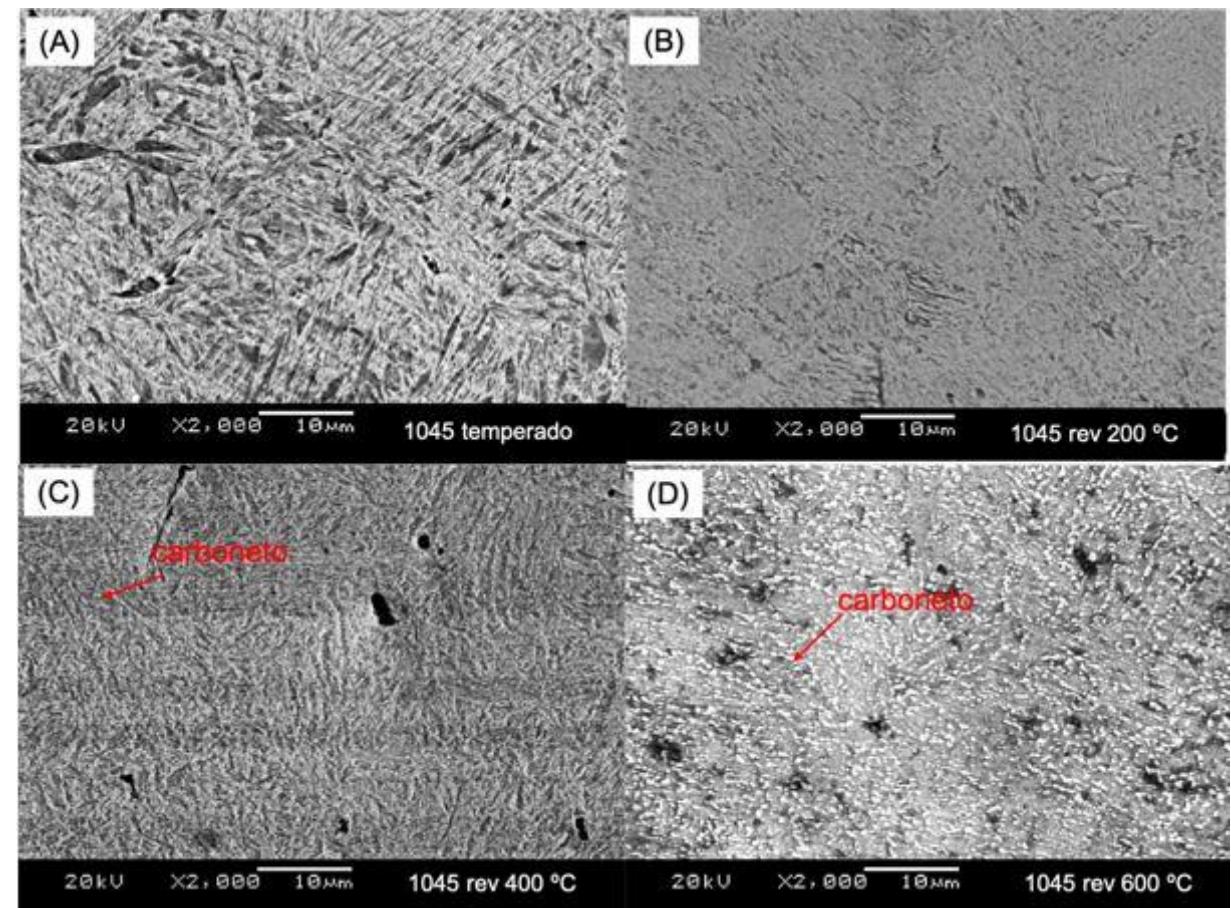

Figura 3: Micrografias aço SAE 1045 como temperado (a), revenido a $200{ }^{\circ} \mathrm{C}$ (b), $400{ }^{\circ} \mathrm{C}$ (c), e $600{ }^{\circ} \mathrm{C}$ (d).

Os valores de dureza obtidos para amostras de aço SAE 1045 revenidas em forno convencional estão apresentados na Tabela 1. Nota-se que conforme previsto na literatura [31, 32], os valores de dureza diminuem conforme a temperatura de revenimento aumenta. Esta diminuição ocorre em função da 
transformação da estrutura martensítica em martensita revenida, conforme observado nas micrografias da Figura 3.

Tabela 1: Dureza média das amostras de aço SAE 1045 revenidas em forno resistivo.

\begin{tabular}{l|l}
\hline TEMPERATURA DE REVENIMENTO CONVENCIONAL AÇO SAE 1045 [ $\left.{ }^{\circ} \mathbf{C}\right]$ & DUREZA MÉDIA [HV] \\
\hline Sem revenimento & $652+28$ \\
\hline 200 & $627+25$ \\
\hline 300 & $520+15$ \\
\hline 400 & $430+20$ \\
\hline 500 & $326+26$ \\
\hline 600 & $263+25$ \\
\hline
\end{tabular}

A microestrutura e os valores de dureza obtidos após o revenimento em forno resistivo das amostras de referência do aço SAE 8640 estão apresentados na Figura 4 e Tabela 2, respectivamente. A microestrutura do aço SAE 8640 temperado em água (Figura 4 a) é composta por martensita com estrutura acicular bem definida com uma pequena quantidade de austenita retida. Durante o revenido a $200^{\circ} \mathrm{C}$ (Figura 4b) praticamente não houve mudanças na microestrutura do aço SAE 8640, apenas uma leve perda da definição estrutura acicular da martensita. Na amostra revenida a $400{ }^{\circ} \mathrm{C}$ (Figura 4c), observa-se uma martensita revenida com uma estrutura acicular menos definida e com a presença de uma pequena quantidade de carbonetos esferoidizados. Como esperado a quantidade de carbonetos esferoidizados aumenta no aço SAE 8640 revenido a $600{ }^{\circ} \mathrm{C}$ (Figura $4 \mathrm{~d}$ ). Logo, nota-se que temperaturas de revenimento maiores resultaram em alterações microestruturais mais significativas no aço SAE 8640, seguindo o comportamento esperado na literatura $[30,32,33]$.

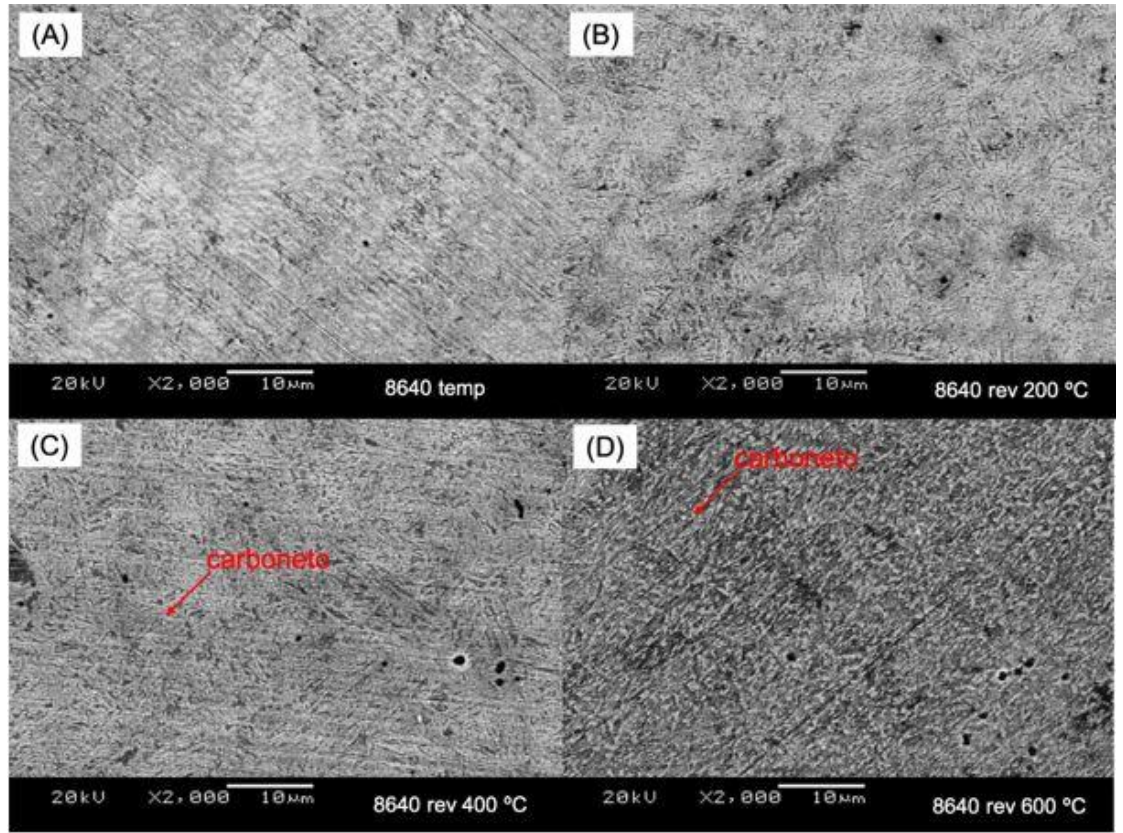

Figura 4: Micrografias aço SAE 8640 como temperado (a), revenido a $200^{\circ} \mathrm{C}$ (b), $400{ }^{\circ} \mathrm{C}(\mathrm{c})$, e $600{ }^{\circ} \mathrm{C}$ (d).

Assim como nas amostras de aço SAE 1045, a dureza média das amostras do aço SAE 8640 também diminuí em função do aumento da temperatura de revenimento, como pode ser observado na Tabela 2 . Além disso destaca-se que não houve variação significativa de dureza entre as amostras revenida a $500^{\circ}$ e a $600{ }^{\circ} \mathrm{C}$, o que está relacionado com o fato de que as principais mudanças microestruturais decorrentes do revenido no aço SAE 8640 ocorrem na faixa entre 300 e $500{ }^{\circ} \mathrm{C}$, e, portanto, a utilização de temperaturas acima deste 
valor resulta apenas num lento aumento no tamanho de grão e consequentemente lenta diminuição no valor de dureza.

Tabela 2: Dureza média das amostras de aço SAE 8640 revenidas em forno resistivo.

\begin{tabular}{c|c}
\hline TEMPERATURA DE REVENIMENTO CONVENCIONAL DO AÇO SAE 8640 [ [ $\mathbf{C}]$ & DUREZA MÉDIA [HV] \\
\hline Sem revenimento & $681+30$ \\
\hline 200 & $628 \pm 23$ \\
\hline 300 & $534 \pm 26$ \\
\hline 400 & $386 \pm 24$ \\
\hline 500 & $285 \pm 23$ \\
\hline 600 & $282 \pm 23$ \\
\hline
\end{tabular}

Em relação aos valores de dureza obtidos para amostras revenidas em plasma, os mesmos são apresentados na Tabela 3. Nota-se que o aumento da corrente causou um aumento na temperatura do plasma e consequentemente uma diminuição da dureza média das amostras.

Tabela 3: Dureza média das amostras de aço SAE 1045 e SAE 8640 revenidas em plasma.

\begin{tabular}{l|c|c|c|c}
\hline Material & $\begin{array}{c}\text { Corrente } \\
{[\mathbf{m A}]}\end{array}$ & $\begin{array}{c}\text { Temperatura do porta- } \\
\text { amostra medida pelo } \\
\text { pirômetro }\left[{ }^{\circ} \mathbf{C}\right]\end{array}$ & $\begin{array}{c}\text { Dureza média } \\
{[\mathbf{H V}]}\end{array}$ & $\begin{array}{c}\text { Temperatura calculada } \\
\text { pelas equações 1 e 2 [ }{ }^{\circ} \mathbf{C} \text { ] }\end{array}$ \\
\hline Aço SAE 1045 & 100 & 260 & $307 \pm 44$ & 511,5 \\
\hline Aço SAE 1045 & 200 & 312 & $219 \pm 46$ & 606,0 \\
\hline Aço SAE 1045 & 300 & 380 & $177 \pm 11$ & 654,1 \\
\hline Aço SAE 8640 & 100 & 290 & $376 \pm 36$ & 410,0 \\
\hline Aço SAE 8640 & 200 & 350 & $309 \pm 28$ & 486,4 \\
\hline Aço SAE 8640 & 300 & 430 & $293 \pm 9$ & 506,7 \\
\hline
\end{tabular}

Observa-se que a dureza das amostras de aço SAE 1045 revenidas diminui aproximadamente $42 \%$ com aumento da corrente de $100 \mathrm{~mA}$ para $200 \mathrm{~mA}$. Enquanto nas amostras de aço SAE 8640, a dureza diminui cerca de $22 \%$. Outro ponto a destacar, é a maior homogeneidade da dureza nas amostras revenidas em correntes mais altas, o que é evidenciado através da diminuição do desvio padrão das medidas de dureza nessas amostras. Diferentemente das amostras revenidas em forno convencional, nas quais o desvio padrão manteve-se praticamente constante, as amostras revenidas em plasma apresentam uma diminuição significativa do desvio padrão com aumento de corrente, ou seja, com o aumento da temperatura. A maior variação dos valores de dureza pode ser relacionada com a presença de picos térmicos, os quais apresentam um efeito mais pronunciado nas amostras revenidas em temperaturas mais baixas. As amostras revenidas em correntes menores são revenidas em temperatura médias menores, de modo que um maior gradiente térmico é esperado entre a região nas quais ocorre o pico térmico e o centro da amostra. E, portanto, há uma maior variação na dureza ocorre nessas amostras.

Ao analisar, a micrografia da amostra de aço SAE 1045, revenida a 100 mA (Figura 5a), observa-se que a microestrutura é composta por martensita revenida similar a amostra revenida em forno resistivo a 400 ${ }^{\circ} \mathrm{C}$, apesar da temperatura no porta-amostra medida no pirômetro indicar apenas $260^{\circ} \mathrm{C}$, o que está de acordo com o valor de dureza de apenas $307 \mathrm{HV}$. Com relação a amostra de aço SAE 1045 revenida utilizando uma corrente de $200 \mathrm{~mA}$ (Figura 5b), nota-se uma dureza de 219 HV e uma microestrutura constituída por uma maior quantidade de carbonetos esferoidizados semelhante a amostra revenida em forno resistivo a $600{ }^{\circ} \mathrm{C}$, todavia a temperatura medida pelo pirômetro foi de $312^{\circ} \mathrm{C}$. Na micrografia da amostra aço SAE 1045 revenida com uma corrente de $300 \mathrm{~mA}$ (Figura 5c), percebe-se que a microestrutura é composta de esferoidita com carbonetos de ferro (cementita) maiores do que da amostra revenida em forno resistivo a 600 ${ }^{\circ} \mathrm{C}$, o que concorda com o valor de dureza de apenas $177 \mathrm{HV}$. 

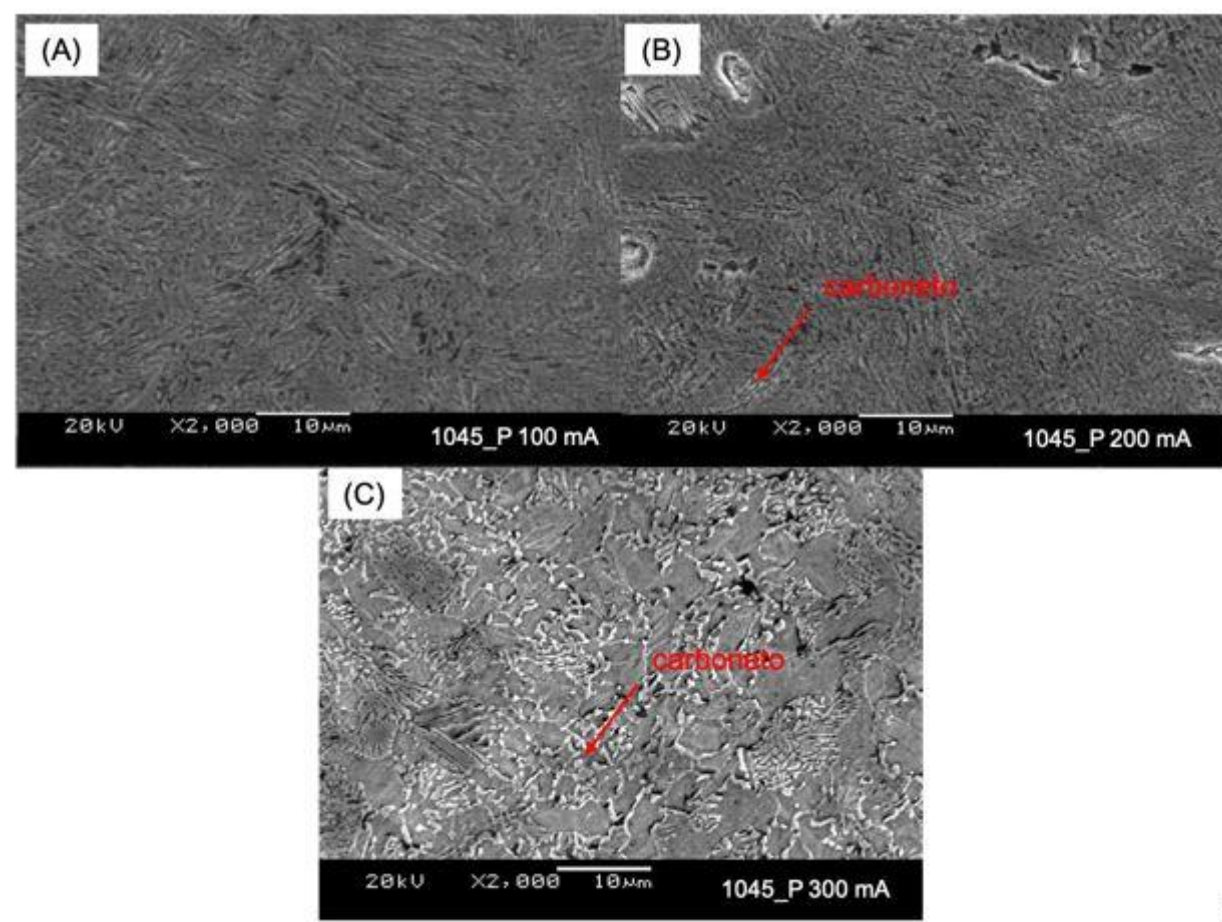

Figura 5: Aço SAE 1045 revenido em plasma utilizando corrente de: (a) $100 \mathrm{~mA}$, (b) $200 \mathrm{~mA}$ e (c) $300 \mathrm{~mA}$.

Em relação ao aço SAE 8640 revenido utilizando uma corrente de 100 mA (Figura 6a), observa-se que a microestrutura é formada por martensita revenida e alguns carbonetos, e uma dureza de $376 \mathrm{HV}$ similar a amostra revenida em forno resistivo a $400{ }^{\circ} \mathrm{C}$, porém a temperatura indicada pelo pirômetro foi de $290{ }^{\circ} \mathrm{C}$. $\mathrm{Na}$ amostra do aço SAE 8640 revenido a $200 \mathrm{~mA}$ (Figura 6b), nota-se novamente que a microestrutura é composta de martensita revenida e carbonetos, sendo que os carbonetos aparecem em maior quantidade. Por fim, a amostra de aço SAE 8640 revenido a $300 \mathrm{~mA}$ (Figura 6c) é composta por martensita revenida com carboneto de ferro esferoidizado e apresentou uma dureza de $294 \mathrm{HV}$, apresentando semelhança com a amostra revenida em forno resistivo a uma temperatura de $600{ }^{\circ} \mathrm{C}$, entretanto a temperatura medida com pirômetro foi $430{ }^{\circ} \mathrm{C}$. 

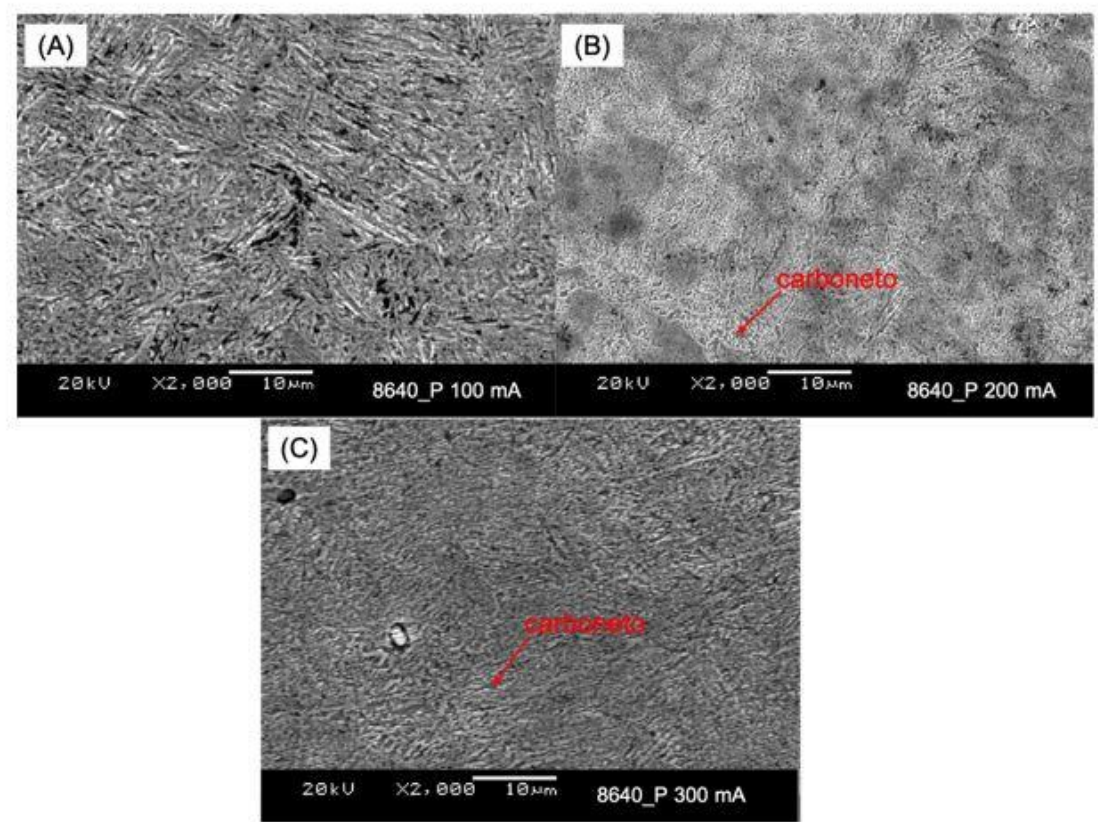

Figura 6: Aço SAE 8640 revenido em plasma utilizando corrente de: (a) $100 \mathrm{~mA}$, (b) $200 \mathrm{~mA}$ e (c) $300 \mathrm{~mA}$.

A fim de avaliar a uniformidade do revenimento, valores de dureza foram coletados ao longo da seção transversal como mostrado na Figura 2. Observou-se que valores de dureza tanto para amostra SAE 8640 como para SAE 1045 mantiveram-se uniforme nas amostras revenidas em forno resistivo (Figura 7), enquanto as amostras revenidas em plasma apresentaram um menor valor de dureza na superfície (Figura 8). Esses menores valores de dureza obtidos na superfície da amostra corroboram com tese da presença de picos térmicos, ou seja, regiões com maior temperatura localizada na superfície das amostras confirmando resultados apresentados previamente na literatura [20, 22, 25].

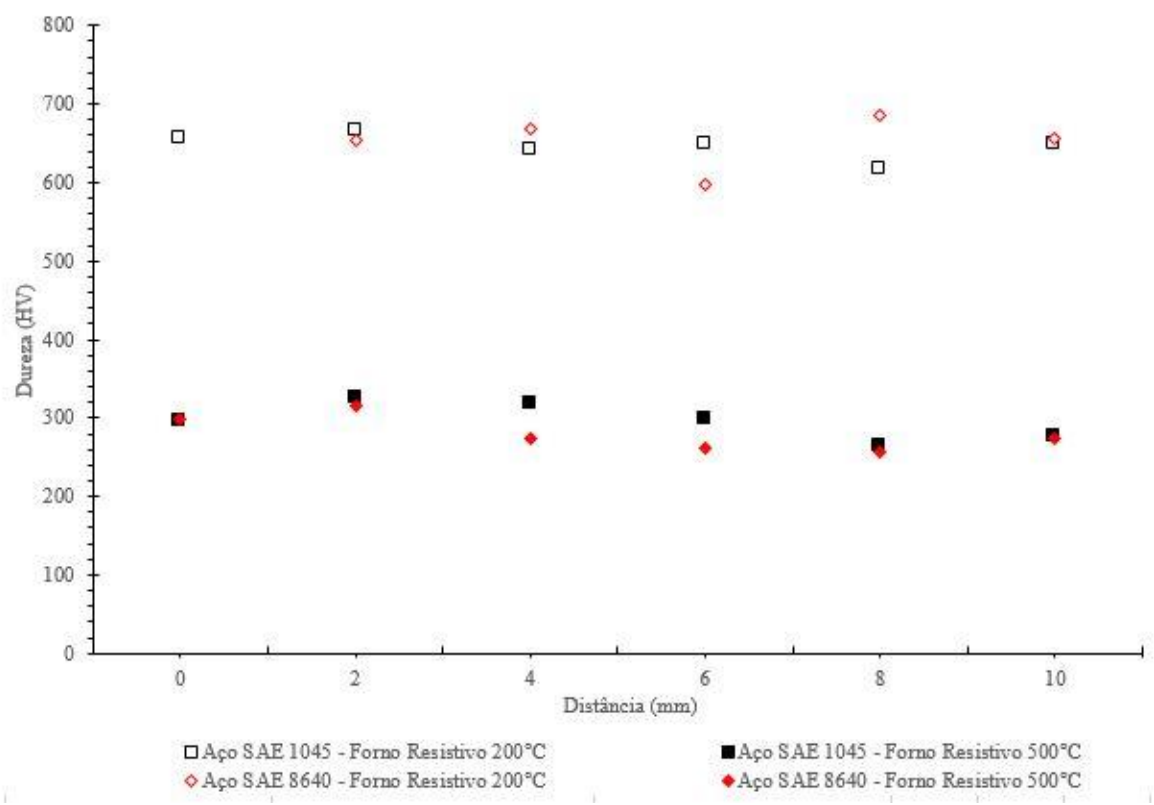

Figura 7: Dureza versus distância para amostras de aço SAE 1045 e SAE 8640 revenidas em Forno Resistivo. 


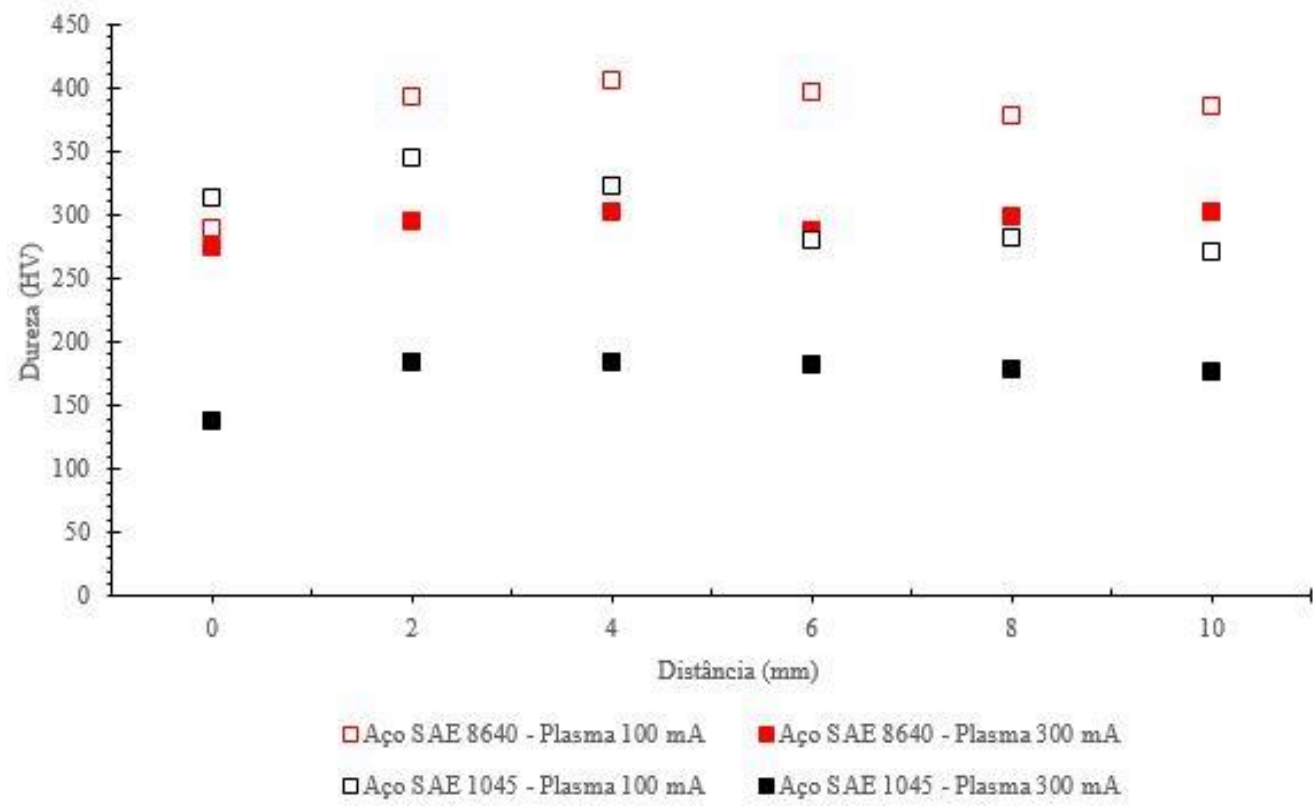

Figura 8: Dureza versus distância para amostras de aço SAE 1045 e SAE 8640 revenidas em plasma.

É importante destacar que ao correlacionar os dados, sobre o revenimento a plasma, obtidos na Tabela 3 , com os dados sobre o revenimento realizado em forno resistivo, informados na Tabela 1 e Tabela 2, notase uma grande variação na dureza média obtida, ao comparar as faixas de temperaturas próximas. Desta maneira, percebe-se que as medições de temperaturas realizadas com o pirômetro no porta-amostra ao final do tratamento não condizem com as temperaturas máximas em que as amostras foram submetidas no processo a plasma. Assim sendo, para determinar a temperatura equivalente das amostras revenidas no plasma, utilizou-se os valores de dureza obtidos no processo de revenimento em forno resistivo como base para a determinação de uma função que relaciona a temperatura e dureza para amostras de aço SAE 1045 e SAE 8640 revenidas no intervalo entre $200^{\circ} \mathrm{C}$ e $600^{\circ} \mathrm{C}$. A partir desta função, foi possível estimar a temperatura equivalente durante o processo de revenimento a plasma, para as amostras de aço SAE 1045 e SAE 8640.

Para o aço SAE 1045 ao relacionar a dureza obtida com sua respectiva temperatura (Figura 9), observa-se que a linha de tendência polinomial se encontra muito próxima dos resultados obtidos no processo realizado em forno resistivo. Assim sendo, a linha de tendência para o aço SAE 1045 foi determinada conforme a Equação 1, que irá permitir que a temperatura de revenimento da amostra seja definida em relação a dureza da amostra.

$$
T=0.0005 * \mathrm{H}^{2}-1.3352 * \mathrm{H}+874.32
$$




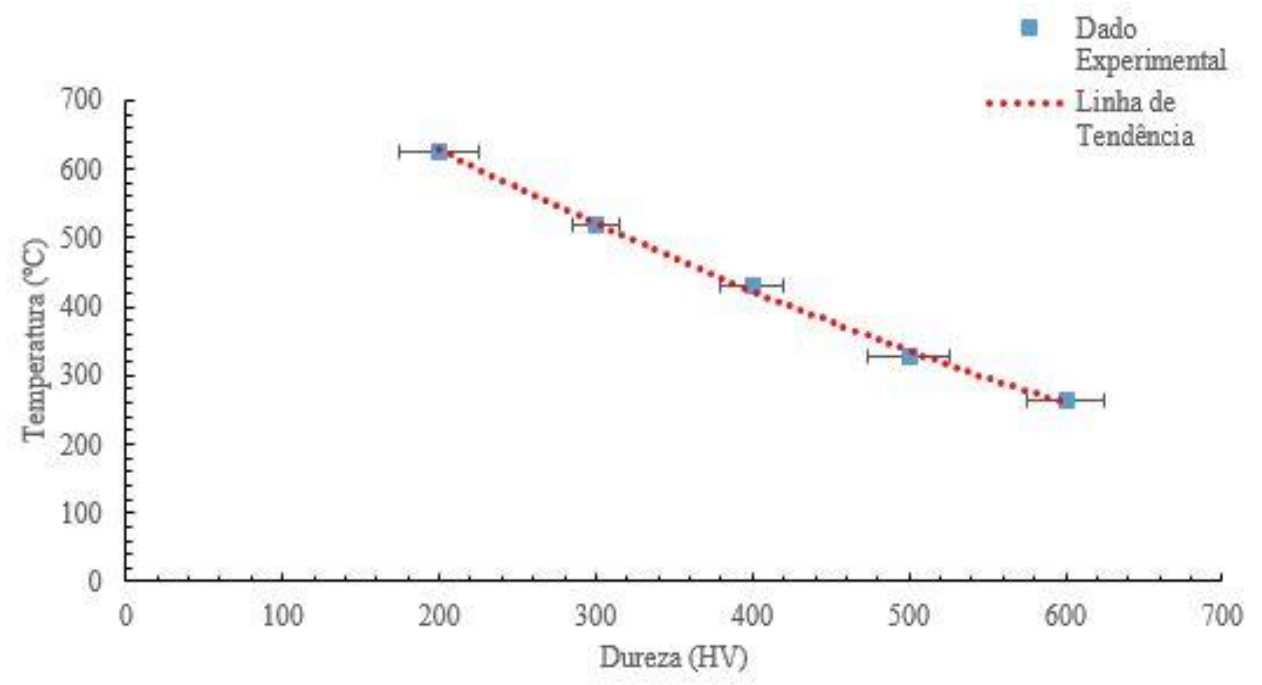

Figura 9: Temperatura versus dureza, mostrando a curva de ajuste polinomial obtida para o aço SAE 1045.

O mesmo procedimento foi aplicado ao aço SAE 8640, como pode ser observado na Figura 10, onde a relação entre a temperatura e dureza é determinada pela equação 2.

$$
T=0.0016 * H^{2}-2.2459 * H+1028
$$

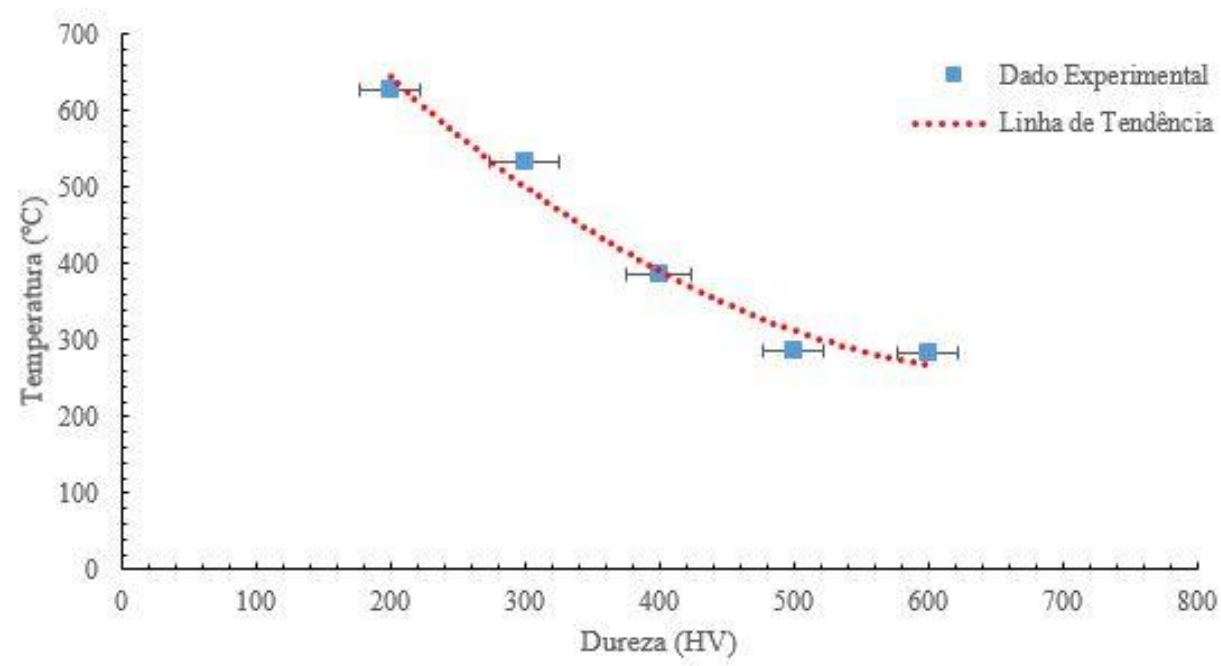

Figura 10: Temperatura versus dureza, mostrando a curva de ajuste polinomial obtida para o aço SAE 8640.

Com a determinação da Equação 1 e da Equação 2, foi possível estimar a temperatura em que as amostras foram expostas durante o processo de revenimento em plasma (Tabela 3). Observa-se que a temperatura equivalente calculada pelas equações (1) e (2) foi significativamente maior que temperatura medida pelo pirômetro. Isso está relacionado com a presença de picos térmicos durante o revenimento em plasma, o que eleva a temperatura em determinados locais da amostra e com as características do processo a plasma como por exemplo a presença de espécies ativas de hidrogênio que agem removendo contaminantes da superfície da amostra e da superfície dos contornos de grão, provavelmente favorecendo o crescimento de grão e a nucleação de novas fases a partir dos contornos de grão, como observado na literatura [23, 24]. Durante o tratamento no plasma, ambos os aços foram submetidos a mesma corrente, todavia as temperaturas calculadas para uma mesma corrente aplicada foram diferentes. Isso, pode ser atribuído as diferentes composições dos aços, que acarretaram como consequência, em diferentes taxas de transferência de calor nas amostras. Notou-se que o aço SAE 8640 apresentou temperaturas menores quando comparado ao aço SAE 1045, o que 
pode estar relacionado a maior presença de elementos de liga, o que reduz seu coeficiente de condutividade térmica e, portanto, sua taxa de transferência de calor da superfície para o centro da amostra.

Observa-se que a medição da temperatura no processo de revenimento a plasma, realizada por meio da utilização de um pirômetro não é precisa, visto que, a temperatura no porta-amostra pode ser significativamente menor do que a temperatura momentânea em um pico térmico na superfície da amostra. Os resultados obtidos corroboram com estudos que citam a presença de gradiente térmicos em amostras de aço AISI M35 aquecidas em plasma $[19,20]$ e que indicam que os processos termicamente ativados são favorecidos no plasma $[23,26]$.

\section{CONCLUSÕES}

A partir da correlação entre a temperatura e a dureza obtida por meio do revenimento realizado em forno resistivo foi possível estimar a temperatura equivalente durante o revenimento em plasma. Notou-se que a temperatura equivalente é consideravelmente maior que a temperatura medida no porta-amostra por um pirômetro. Além disso, a presença de picos térmicos foi observada em ambos os aços SAE 1045 e SAE 8640, o que resultou em uma diminuição da dureza na superfície das amostras. Além disso, os resultados obtidos indicam que o aquecimento por plasma favorece os processos termicamente ativados, facilitando a transformação de fases e crescimento dos carbonetos de ferro. É importante destacar que a temperatura das amostras aquecidas no plasma, em condições iguais de corrente e pressão, pode variar de acordo com a composição do material. Portanto, justifica-se a necessidade de investigar o aquecimento de diferentes materiais em plasma.

Através deste trabalho foi possível melhorar a compreensão acerca dos mecanismos de transferência de calor em um reator de nitretação iônica e confirmar a presença de picos térmicos e gradientes de temperatura em amostras de aço. Os resultados obtidos nesse trabalho poderão ser aplicados aos processos de nitretação iônica, uma vez que conhecer a temperatura é chave para o ajuste da microestrutura e consequentemente das propriedades mecânicas dos aços.

\section{REFERÊNCIAS}

[1] LIEBERMAN, M.A., LICHTENBERG, A.J., Principles of plasma discharges and materials processing, 2 ed., New Jersey, John Wiley \&Sons, 2005.

[2] MANDOLFINO, C., "Polypropylene surface modification by low pressure plasma to increase adhesive bonding: Effect of process parameters", Surface and coatings technology, v. 366, n. 25, pp. 331-337, May. 2019.

[3] ANJOS, A.D., SCHEUER, C.J., BRUNATTO, S.F., et al., "Low-temperature plasma nitrocarburizing of the AISI 420 martensite stainless steel: microstructure and process kinetics", Surface and Coatings Technology, v. 275, n. 15, pp. 51-57, Aug. 2015.

[4] SHUSKUS, A.J., COWHER, M.E., Plasma enhanced deposition of semiconductors, U.S, Patent No. 4,421,592, 20 Dec. 1983.

[5] ZHAO, G.H., AUNE, R.E., ESPALLAGAS, N., "Tribocorrosion studies of metallic biomaterials: The effect of plasma nitriding and DLC surface modifications", Journal of the mechanical behavior of biomedical materials, v. 63, pp. 100-114, Oct. 2016.

[6] SUN, X., YANG, X., XIANG, H., et al., "Nitrogen-doped CoOx/carbon nanotubes derived by plasmaenhanced atomic layer deposition: Efficient bifunctional electrocatalyst for oxygen reduction and evolution reactions", Electrochimica Acta, v. 296, n. 10, pp. 964-971, Feb. 2019.

[7] USMAN, C.A., MANSOOR, B., MUNGOLE, T., et al., "Corrosion mechanism in PVD deposited nanoscale titanium nitride thin film with intercalated titanium for protecting the surface of silicon", Electrochimica Acta, v. 264, pp. 69-82, Jan. 2018.

[8] ZOLFAGHARI, P., KHALEDIAN, H.R., ALIASGHARLOU, N., et al., "Facile surface modification of immobilized rutile nanoparticles by non-thermal glow discharge plasma: Effect of treatment gases on photocatalytic process", Applied Surface Science, vol. 490, n. 1, pg. 266-277, Oct. 2019.

[9] RATZKER, B., WAGNER, A., SOKOL, M., et al., "Optical and mechanical properties of transparent alumina fabricated by high-pressure spark plasma sintering", Journal of the European Ceramic Society, v. 39, n. 8, pg. 2712-2719, Jul. 2019.

[10] KERSTEN, H., DEUTSCH, H., STEFFEN, H., et al., "The energy balance at substrate surfaces during plasma processing", Vacuum, v. 63, n. 3, pp. 385-431, Jul. 2001. 
[11] PINEDO, C.E., “Tratamentos superficiais para aplicações tribológicas”, Metalurgia e Materiais, v. 60, n. 543, pp. 162-164, Abril. 2004.

[12] CIROCKA, A., ZARZECZAŃSKA, D., WCISŁO, A., et al., "Tuning of the Electrochemical Properties of Transparent Fluorine-doped Tin Oxide Electrodes by Microwave Pulsed-plasma Polymerized Allylamine", Electrochimica Acta, v.313, n. 1, pp. 432-440, Aug. 2019.

[13] BÖTEL, F., ZIMMERMANN, T., SÜTEL, M., et al., "Influence of different low-pressure plasma process parameters on shear bond strength between veneering composites and PEEK materials", Dental Materials, v. 34, n. 9, pp. 246-254, Sep. 2018.

[14] DE GEYTER, N., MORENT, R., VAN VLIERBERGHE, S., et al., "Effect of electrode geometry on the uniformity of plasma-polymerized methyl methacrylate coatings", Progress in Organic Coatings, v. 70, n. 4, pp. 293-299, Apr. 2011.

[15] FAN, X., DARUT, G., PLANCHE, M.P., et al., "Preparation and characterization of aluminum-based coatings deposited by very low-pressure plasma spray", Surface and Coatings Technology, v. 380, n. 25, 125034, Dec. 2019.

[16] ALVES JUNIOR, C., HAJEK, V., SANTOS, C., "Thermal behavior of supersolidus bronze powder compacts during heating by hollow cathode discharge", Materials Science and Engineering A-structural Materials Properties Microstructure and Processing, v. 348, n. 1-2, pp. 84-89, May. 2003.

[17] AKAMATSU, H., YATSUZUKA, M., "Simulation of surface temperature of metals irradiated by intense pulsed electron, ion and laser beams", Surface and Coatings Technology, v. 169-170, n. 2, pp. 219222, Jun. 2003.

[18] KERSTEN, H., STEFFEN, H., VENDER, D., et al., "On the ion energy transfer to the substrate during titanium deposition in a hollow cathode arc discharge”, Vacuum, v. 46, n. 3, pp. 305-308, Mar. 1995.

[19] LEAL, E.A.D., SOUZA, S.I.S., ALVES, J.C., "Novo método para determinação da temperatura de corpos imersos em plasma”, Revista Brasileira de Aplicações de Vácuo, v. 34, n. 1, pp. 24-28, Feb. 2015.

[20] GALVÃO, N.K.A.M., COSTA, B.L.S., MENDES, M.W.D., et al., "Structural modifications of M35 steel submitted to thermal gradients in plasma reactor", Journal of materials processing technology, v. 200, n. 1-3, pp. 115-119, May 2008.

[21] KRATSCHMER, W., FOSTIROPOULOS, L., HUFFMAN, K., "Solid $\mathrm{C}_{60}$ : A new form of carbon", Nature, v.347, n.33, pp. 354-357, Sep. 1990.

[22] LIMA, J.A., ALVES JR, C., DOS SANTOS, C.A., “Estudo do Gradiente Térmico no Processo de Nitretação a Plasma", Revista Matéria, v.10, n.2, pp 273-283,2005.

[23] PAVANATI, H.C., STRAFFELINI, G., MALISKA, A.M., et al.,"Microstructural and mechanical characterization of iron samples sintered in DC plasma", Materials Science and Engineering A, v.474, pp.12-23,2008.

[24] MALISKA, A.M., PAVANATI, H.C., KLEIN, A.N., et al., "The influence of ion energy bombardment on the surface porosity of plasma sintered iron", Materials Science and Engineering A, v.352,pp 273-278, 2003.

[25] SINKA, V., ALVES JR, C., JANÁK, G., "Plasma sintering of Cu90Sn10 bronze”, Journal of Materials Science Letters, v.21, pp 427-429,2002.

[26] DAUDT, N.F., BRAM, A.P., BARBOSA, A.P.C., et al., "Manufacturing of highly porous titanium by metal injection molding in combination with plasma treatment ", Journal of Materials Processing Technology, v. 239, pp. 202-209, Jan. 2017.

[27] REINHOLD, I., HENDRIKS, C.E. ECKARDT, R., et al., “Argon plasma sintering of inkjet printed silver tracks on polymer", Journal of Materials Chemistry, Bd.19, pp 3384-3388,2009.

[28] GALVÃO, N.K.A.M., Influência da técnica de plasma na obtenção de titânio poroso, Natal: Doctoral thesis, Universidade Federal do Rio Grande do Norte, 2011.

[29] ASSOCIAÇÃO BRASILEIRA DE NORMAS TÉCNICAS. NBR ISO 6507-1: Materiais metálicos Ensaio de dureza Vickers - Parte 1: Método de ensaio. Rio de Janeiro, p. 30, 2019.

[30] COSTA e SILVA, A.L., MEI, P.R., Aços e ligas especiais, 3 ed, São Paulo, Edgard Blucher, 2010.

[31] COLPAERT, H., Metalografia dos produtos siderúrgicos comuns, 4 ed, São Paulo, Edgard Blucher, 2008. 
[32] KRAUSS, G., Steels: heat treatment and processing principles, Materials Park, OH: ASM international, 1990.

[33] BHADESHIA, H., HONEYCOMBE ,R., Steels: Microstructure and Properties, 4th ed, Elsevier, 2017.

\section{ORCID}

Otávio Henrique de Andrade Disconzi

Diego Michael Cornelius dos Santos

Bruno Cuchi Bordignon

Júlia Beltrame Bisogno

Inácio da Fontoura Limberger

Natália de Freitas Daudt https://orcid.org/0000-0002-6815-5493

https://orcid.org/0000-0003-2776-592X

https://orcid.org/0000-0001-8771-8880

https://orcid.org/0000-0002-8178-2889

https://orcid.org/0000-0002-8089-6032

https://orcid.org/0000-0001-8028-9112 